\title{
https://doi.org/10.48009/1_iis_2005_101-106 \\ INFORMATION SYSTEMS AND CONTINUOUS LEARNING THROUGH AN ALTERNATIVE TO BRAILLE
}

\author{
Elia Chepaitis, Fairfield University, echepaitis@mail.fairfield.edu
}

\begin{abstract}
From the emergence of the World Wide Web through Y2K and beyond, the scope of Information Systems (IS) has expanded beyond intra-organizational goals and interests, into areas that affect the common good. This paper describes ongoing research to improve continuous learning for the visually impaired. The author presents an easy-to-use, ASCIIcompatible, patented and tested alternative to Braille as the foundation for a new information infrastructure for the blind using mainstream software, and hardware with slight alterations. Using this easy-to-learn language as an interface, open and seamless ICT architecture can revolutionize the content and context of information access for the blind. The author includes a brief literature search, and hypothesizes that Braille's monopoly as the supported language for persons with vision problems is not a technical, but a political and cultural phenomenon.
\end{abstract}

Keywords: alternative to Braille, tactile language, information infrastructure

\section{INTRODUCTION}

The widening scope of Information Systems (IS) increasingly extends into is areas that involve the general good: security, the protection of intellectual property, the health of infrastructures, economic development, and ethics. The first page of the latest Sprague and McNurlin textbook opens with a proclamation: "Information technology (IT) - computers and telecommunicationsis having the kind of revolutionary, restructuring impact that has been expected and touted for years [2006]." In the last decade, more people used computers for communication than computation. Networked information and communication technologies (ICTs) create vast opportunities for lifelong learning and distance education. However, in the networked economy, best practices for continuous learning embrace multidisciplinary strategies and skills, and build upon existing user skills and preferences for optimal information systems and efficacy. A circle of stakeholders, including ICT and other professionals, are developing opportunities for continuous learning for heterogeneous readers: using minority languages, living in varied infrastructures, constrained by political and economic systems, or isolated by physical disabilities.

This article focuses on one population in all these overlapping groups--adults who lose vision. The visually impaired have been restricted in language choices, in the access to real-time and customized information, and in the ability to share documents easily with sighted partners up to the present. A new language promises to be the bridge to continued learning for this population. First, this article describes results of testing the alternative to Braille at the State University of New York School of Optometry, and second, examines Braille as a historic and meaningful anomaly. 


\section{An Alternative to Braille: Elementary Imprint Assistance (ELIA)}

The opportunity to design a new information infrastructure for the newly visually impaired provides 1) an easy migration from sighted literacy and 2) a seamless interface with contemporary information and communication technologies (ICTs). The monolingual dominance of Braille, a language that physically bears no resemblance to the familiar alphabet whose shapes adults recognize and anticipate since childhood, violates major tenets of industrial engineering, particularly panel and display theory. However, no alternate easy-to-learn and easy-to-use languages have been tolerated.

There are 1.8 million severely visually impaired adults in the United States, among whom only 59,000 use Braille, the only language supported by the associations for the blind for more than a century. Readers who lose vision lose not only access to networked information, work, and community, but also advantages of literacy itself-from the ability to read labels on prescriptions to the tools to review voice transcripts from a Kurzweil reader. An easy-to-use, ASCIIcompatible, patented and tested alternative to Braille, Elementary Imprint Assistance or ELIA, is the foundation for a new information infrastructure for the blind (Figure 1)[10]. Using this easyto-learn language as an interface, open and seamless ICT architecture can revolutionize the content and context of information access, especially for adults who lose vision.

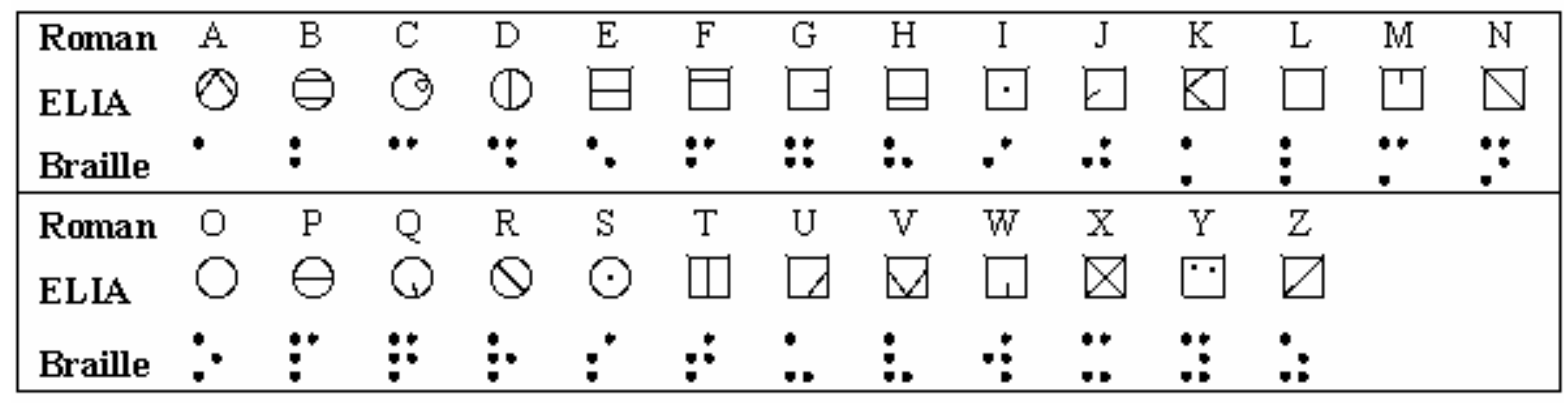

Figure 1. Font Designs Compared

Under private foundation grants from 2001 to 2004 and a National Institute of Health grant in 2005 , researchers taught and tested groups of as many as one hundred visually impaired subjects with either Braille or ELIA. ELIA proved to be substantially easier to learn and feel than Braille or the standard Roman alphabet for previously sighted readers (Figure 2).

In 2004, at letter heights of $0.7 \mathrm{~cm}$ (standard Braille size), $0.9 \mathrm{~cm}, 1.1 \mathrm{~cm}$ and $1.3 \mathrm{~cm}$, the ELIA font offered greater tangibility than Braille $(\mathrm{p}<0.016)$. At present, based on serendipitous discoveries during the 2004 trials, researchers are examining to what extent scalability and letter spacing at different font sizes can enhance self-learning and reading speeds even further. Testing materials in both Braille and ELIA were produced with a Tektronix Phaser 300 wax jet printer configured to overprint images until the font height was sufficient for tactile recognition. The 2004 subjects participated in 1.5 hour sessions, four times a week over ten weeks, for a total of 60 hours of training. The characters per minute $(\mathrm{cpm})(\mathrm{p}<0.01)$ and words per minute $(\mathrm{wpm})$ $(p<0.016)$ reading speeds of the ELIA group were twice that of the Braille group in letter, 
sentence, and random reading tests—at every font size, letter spacing, or raise (height) in terms of score, speed, and correct words per minute. There was, as expected, a correlation between age and tactile acuity that affected subjects significantly at $0.7 \mathrm{~cm}$ (standard Braille size).

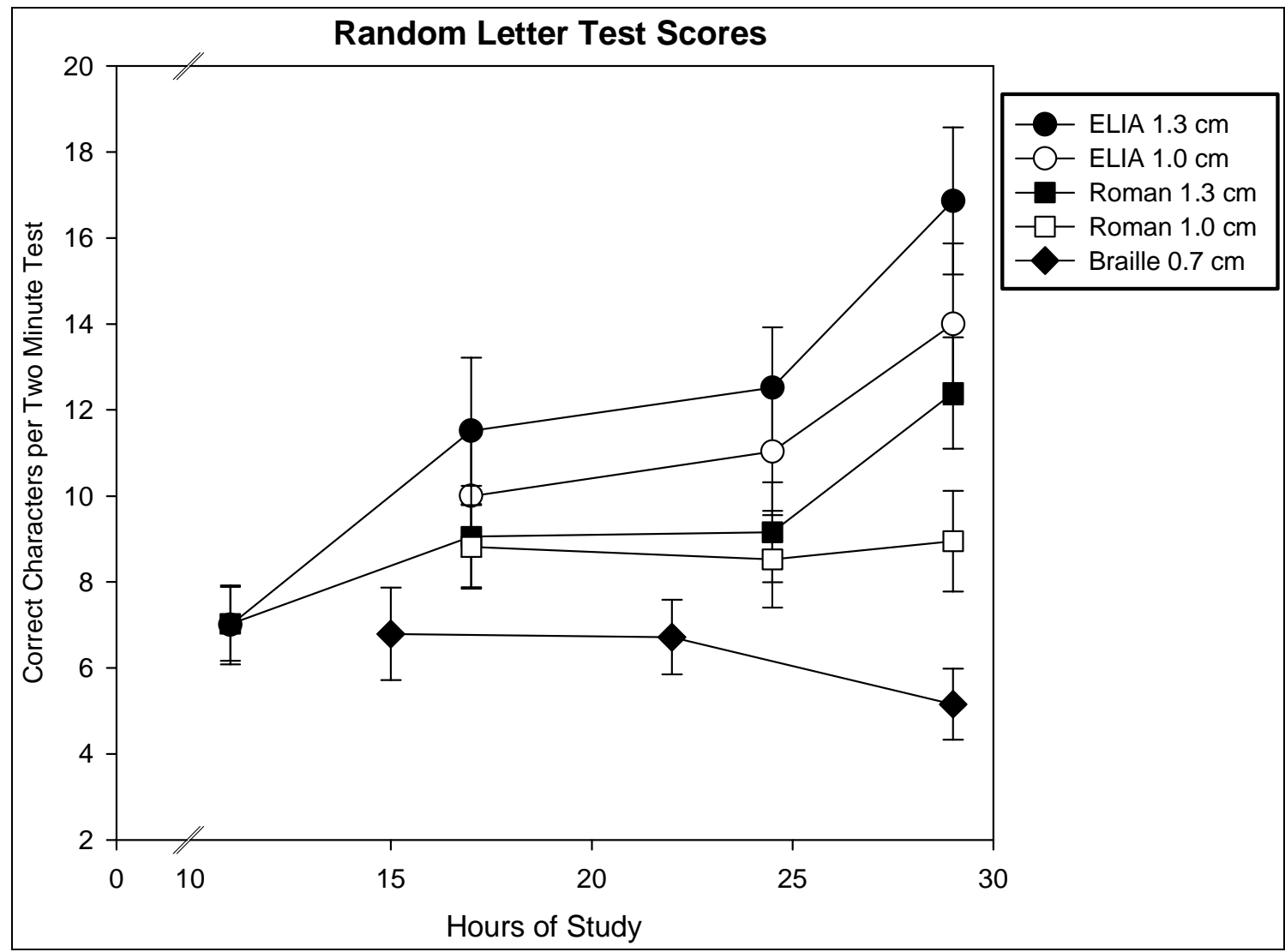

Figure 2. SUNY Test Results, 2004 [1]

\section{A Nascent Information Infrastructure for Continued Learning}

The new alphabet was designed using mainstream human engineering principles and, consequently, resembles the Roman alphabet, with two types of frames to distinguish one letter from another, and for ease of navigation [4]. Its greatest advantage is that readers who have lost vision can learn to recognize and feel characters in a matter of hours versus the months or years it takes to recognize Braille. Reading proficiency can be achieved in weeks, especially because ELIA fonts are scalable and can be gradually reduced in size as acumen increases.

The content and the context of information for the blind can be radically improved, including the ability to scan, print, and read a daily headline, to correspond via e-mail, or to access vast stores of reference material. Output will be available on inexpensive imprinters with a one-on-one correspondence with the regular alphabet, unlike Braille; and input devices feature raised keyboard symbols, as well as voice, scanners, and low-tech labelers. No special software is needed, since the alphabet and numbers, and punctuation have a one-on-one correspondence with the existing Roman alphabet, unlike Braille. 
In time, hopefully, a tactile computer screen can be used with the ELIA font. Combined with electronic text and personal digital technology, a tactile renewable display can create a portable e-book, the user can access digital material and be spared the effort of carrying around bulky paper texts presently used for tactile reading.

\section{Braille as a Legacy System: The Impact of Culture on Technology}

Braille is a nineteenth century technology, adapted from the Napoleonic system for sending short messages at night. The user base declined precipitously as the blind population tends to lose vision at an older age and as schools for the blind were rejected in favor of mainstream education. However, Braille continues to be offered as the standard language of choice although years of study are necessary to become proficient, and although the majority of the newly blind-older, previously sighted adults—rarely can master Braille.

Although Braille is used by less than $8 \%$ of the blind, and does not provide a robust interface with state-of -the-art information and communication technologies (ICTs), it has been the exclusive language supported by leading NGO advocates for the blind since the nineteenth century. In fact, these organizations opposed books on tape initially because of fear that fewer persons would rely on Braille [3]. The acceptance of Braille as the only accepted language for the blind by agencies such as the National Federation for the Blind (NFB) and the American Foundation for the Blind (AFB) raises issues of fairness and efficiency across several disciplines, not only IS. For persons who lose vision after the age of 21, Braille is extremely difficult to learn. For the majority of the blind population that was previously sighted, one major reason why Braille is difficult to learn is because it is not representative of the traditional Roman alphabet which is "mentally imprinted" on former sighted readers [Millar, 1994]. 99.1\% of the visually impaired are previously sighted and learned the Roman alphabet as their first alphabet. Learning Braille requires months or years of study because it is not similar to the traditional alphabet and because it is difficult to feel tactilely.

Consequently, of the 900,000 who lose significant vision every year, there are less than 500 new Braille readers each year. For those who learn Braille (most who attempt to learn it fail), Braille requires extensive education. Although Braille was a godsend when it was invented, Braille learning and reading is expensive and an unrealistic goal for the vast majority of visually impaired people and family members.

\section{LITERATURE REVIEW}

The research on alternatives to Braille is sparse, possibly because only one language for the blind was supported by the AFB and NFB. E. Chepaitis published a brief description of Elementary Imprint Assistance in a special issue on literacy in The Journal of Visual Impairment and Blindness in 1996 [2]. A proponent of Braille, Susanna Millar published an influential monograph, Understanding and Representing Space: Theory and Evidence from Studies with Blind and Sighted Children in 1994 which has valuable material on teaching the visually impaired by associating symbols with experiences [5]. 
A. Chepaitis, A. et al. summarizes ELIA test results at the State University of New York (SUNY) College of Optometry in "Evaluation of Tactile Fonts for Use by Elderly Visually Impaired Population" in a 2004 article in the Visual Impairment Research., published by the International Society for Low Vision Research and Rehabilitation [1]. The classic Human Factors in Engineering and Design by McCormick and Sanders continues to be of enormous help in the creation of an alternative to Braille, especially for ergonomic principles in the chapter on signals and panel displays [4].

\section{CONCLUSION}

The major obstacles to improved reading systems for adults who lose vision have been culturalnot technological. Turning Neil Postman's Technopoly [1993] on its head, Braille is an example of culture driving technology. This exception fits few patterns. C.P. Snow's Two Cultures [1955] spoke of an unbridgeable divide between technologists and layman that has been increasingly breached by user-friendly, democratic ICTs-but Braille has not been promoted by technologists, but by lay advocates. If Braille were computer software, would it have retained its monopoly?

Inhibiting factors which strike the researcher initially are: a patronizing view toward the visually impaired, the power of a powerful minority to impose language preferences on the majority, the popular misconception that Braille serves the blind well, and the tendency of bureaucracies to perpetuate themselves. The curious lack of an egalitarian and democratic approach to systems design for the blind raises questions. Why is this group expected to learn and adhere to a nonevolving and arcane technology that is nearly two hundred years old? Why are they expected to be exceptional to be able to read? The author hypothesizes that the major obstacles to change have been cultural — not technological. Inhibiting historic factors included: a patronizing view toward the visually impaired, the power of a powerful minority to impose language preferences on the majority, and the lack of leveraged, responsible, and knowledgeable professionals to suggest alternatives.

The emergence of a new tactile language and a new era in life-long learning coincides with other trends. These include: the cost-effective digital society, the aging of populations in advanced economies, the health and vigor of many adults into their advanced years, and the proliferation of opportunities for knowledge workers of every age--across time and distance. In addition, the emergence of advanced technologies: advanced voice recognition, renewable displays, imprinters, and seamless networks will improve not only telecommuting and tele-learning but also the quality of life for adults with vision problems.

The number of Braille readers, always modest, plummeted in the past generation and the decline has accelerated with two trends: the mainstreaming of education for the young who are blind, and accelerating demographic shifts that have created a majority who lose their vision as adults. Braille provides a curious exception to technology transfer patterns, and also to political, intellectual and cultural trends. ICTs in this decade have become democratized: easy to use, customized, and accessible to a massive and diverse user population. Not only are ICTs scalable, adaptable, and available, they are also increasingly powerful and affordable. Electronic 
communication devices are ubiquitous, and computing power has increased exponentially, and prices have steadily declined. It is almost a truism that ITC options change in response to both market [pull] factors and to technological innovation [push] factors. Pull factors include political priorities, demographics, economic development, appropriate infrastructures, and a critical mass of informed professional and public opinion. Both push and pull factors threaten Braille's monopoly.

Research topics in the management of technology (MoT) often focus on themes such as: technology driving culture, the need for adaptation strategies, the pace of acceptance, and socioeconomic displacement. Braille is an anachronism and a singular example of culture driving both technology and also the learning infrastructure for the persons with vision problems. Particularly in distance education and learning, the unquestioned dominance and support of Braille is a critical impediment to the utilization of networked resources that support interactive communications, e-government, m-commerce, real time news, and virtual communities. Braille is an unusual example of the consequences of paternalism and rigid stereotypes in a traditional learning regime, an anomaly that can be altered.

\section{REFERENCES}

1. Chepaitis, A. et al. (2004), “Evaluation of Tactile Fonts for Use by Elderly Visually Impaired Population." Visual Impairment Research. International Society for Low Vision Research and Rehabilitation (ISLVRR). Swets and Zeitlinger, 6(2-3), 111-134.

2. Chepaitis, E. (May-June,1996).An Alternative to Braille: Toward Seamless Information Technologies for Persons with Visual Disabilities. Journal of Visual Impairment and Blindness, 90(3), 86-87.

3. Koestler, F.A. (1976). A Social History of Blindness in America. N.Y.: David McKay Co.

4. McCormick, E.J. \& Sanders, M.S. (1989), Human Factors in Engineering and Design. McGraw-Hill.

5. Millar, S. (1994). Understanding and Representing space: Theory and Evidence from Studies with Blind and Sighted Children. Oxford, England: Clarendon Press

6. Postman, N. (1993). Technopoly: The Surrender of Culture to Technology. N.Y.: Vintage Books.

7. Leonard, R. M.A. (1996). Statistics on Vision Impairment: A Resource Manual, The Lighthouse Inc.

8. Snow, C.P. (1955). The Two Cultures and the Scientific Revolution. N.Y.: Cambridge University Press.

9. Sprague, R. \& McNurlin, B.C. (2006). Information Systems Management in Practice. $\left(7^{\text {th }}\right.$ ed.) NY: Prentice Hall.

10. U. S. Patent Office. Elementary Imprint Assistance, an Alternative to Braille.

11. U.S. Utility Patent \# 041,372, 1988.

12. U. S. Patent Office. U.S. Patent \# Des. 321,903. ,1988.

13. U. S. Patent Office \#Des. 472, 265. 2003. Tactile Font.

14. U. S. Patent Office \#Des.480,750. 2004. Tactile Font. 